\title{
Compostos bioativos e atividade antioxidante de frutos de genótipos de cajá- mangueira
}

\section{Bioactive compounds and antioxidant activity of golden apple fruit genotypes}

\author{
José Roberto Chaves Neto \\ Universidade Federal de Santa Maria \\ E-mail: jose.chavesneto@gmail.com \\ OrcID: https://orcid.org/0000-0003-1799-4630
}

\begin{abstract}
Ana Lima Dantas
Faculdade Nova Esperança

E-mail: dantas.ana.lima@gmail.com

OrcID: https://orcid.org/0000-0002-0572-8274

Ana Paula Pereira Schunemann Universidade Federal de Santa Catarina

E-mail: anaschunemann@gmail.com OrcID: https://orcid.org/0000-0003-0116-2821
\end{abstract}

Luana Ferreira dos Santos

Universidade Federal da Paraíba

E-mail: sferreira.Iuana@gmail.com

OrcID: https://orcid.org/0000-0002-3805-654X

Renato Lima Dantas

Faculdade Nova Esperança

E-mail: renato dantas@hotmail.com.br

OrclD: $\underline{\text { https://orcid.org/0000-0002-5464-9476 }}$

Silvanda de Melo Silva

Universidade Federal da Paraíba

E-mail: silvandisilva@gmail.com

OrcID: https://orcid.org/0000-0003-2106-6458

Resumo: Alguns frutos de plantas do gênero Spondias são apreciados pelos aspectos de sabor e aparência atrativos. Contudo, durante a maturação ocorrem mudanças acentuadas que modificam a qualidade, sobretudo nessas plantas que não são plenamente domesticadas. Por terem frutos ricos em compostos antioxidantes, a pesquisa com tais frutos tem focado em compreender as mudanças que estes apresentam na maturação. Este trabalho teve como objetivo avaliar as mudanças nos compostos bioativos e na atividade antioxidante ocorridas durante a maturação de frutos de genótipos de cajá-mangueira (Spondias cytherea Sonn.). Os frutos provenientes de cinco genótipos (P1, P2, P3, P4 P5) de ocorrência espontânea no estado da Paraíba foram colhidos nos seguintes estádios de maturação: totalmente verde (TV), início de pigmentação amarela (IP), amarelo esverdeado (AE) e predominantemente amarelo (PA). O experimento foi conduzido em delineamento inteiramente casualizado, em esquema fatorial $5 \times 4$, sendo cinco genótipos e quatro estádios de maturação, em seis repetições. A polpa dos frutos foi avaliada quanto ao conteúdo de ácido ascórbico, carotenoides totais, flavonoides amarelos, antocianinas, polifenóis extraíveis totais (PET) e atividade antioxidante total (AAT). Os frutos da cajá-mangueira apresentaram aumento no conteúdo de carotenoides e antocianinas da polpa, redução nos PET e flavonoides amarelos e aumento da AAT ao final da maturação. A atividade antioxidante do cajá-manga aumentou ao final da maturação. O genótipo P3 destacou-se entre os demais por apresentar maior conteúdo de carotenoides totais e flavonoides amarelos ao final da maturação (PA), enquanto os genótipos P1 e P5 por apresentarem maior capacidade antioxidante.

Palavras-chave: Capacidade antioxidante. Compostos fenólicos. Radical ABTS. Spondias cytherea Sonn. 
Abstract: Plants of the genus Spondias have fruits that are appreciated because their attractive taste and appearance. However, marked changes that modify the quality happen during maturation, especially in those plants that are not fully domesticated. Because they have fruits rich in antioxidant compounds, the research about these fruits has focused on understanding the changes they present in maturation. The objective of this study was to evaluate changes in bioactive compounds and antioxidant activity during maturation of different genotypes (P1, P2, P3, P4 and P5) of golden apple (Spondias cytherea Sonn.) fruits. Fruits, from five genotypes of spontaneous occurrence in Paraíba State, were harvested at following maturation stages: fully green (TV), yellow pigmentation (IP), greenish yellow (AE) and predominantly yellow (PA). Experiment was conducted in a completely randomized design, in a $5 \times 4$ factorial scheme, being five genotypes and four maturation stages, in six replicates. The fruit pulp was evaluated for ascorbic acid content, total carotenoids, yellow flavonoids, anthocyanins, total extractable polyphenols (PET) and total antioxidant activity (AAT). Fruits of golden apple presented increase in pulp carotenoid and anthocyanin contents, while reduction in PET and yellow flavonoids, with increase of AAT at the end of maturation. The antioxidant activity of golden apple an increased at the end of maturation. P3 genotype was characterized by a higher content of total carotenoids and yellow flavonoids at the end of maturation (PA), while genotypes P1 and P5 presented a higher antioxidant capacity.

Keywords: Antioxidant capacity. ABTS. Phenolic compounds. Spondias cytherea Sonn.

Data de recebimento: $11 / 10 / 2018$

Data de aprovação: 22/01/2020

\section{Introdução}

DOI: https://doi.org/10.30612/agrarian.v14i52.8924

As regiões Norte e Nordeste apresentam uma grande variedade de frutíferas nativas e exóticas não tradicionais. Dentre estas espécies, destacam-se, em especial, as pertencentes ao gênero Spondias, que são consideradas frutas "potenciais" devido à presença de compostos bioativos reconhecidos por proporcionar benefícios à saúde (Fonseca et al., 2017). Devido a isso e a busca por parte dos consumidores por alimentos funcionais, estes frutos ao longo do tempo vêm apresentando um maior valor agregado (Neves, Tosin, Benedette \& Cisneros-Zevallos, 2015; Dantas, Silva, Dantas, Sousa \& Schunemann, 2016).

Dentre as frutíferas pertencentes a esse gênero, destaca-se a Spondias cytherea Sonn. (Cajámangueira), originária da região da Indo-Malásia ao Taiti que se expandiu para as outras regiões tropicais (Koubala, Kansci \& Ralet, 2018). No Brasil, está distribuída na zona da mata, no agreste, nas regiões semiáridas, subúmida e semiúmida do nordeste brasileiro (Lorenzi, Bacher, Lacerda \& Sartori, 2006) e, no Estado da Paraíba, é encontrada na mesorregião do Brejo Paraibano e Mata Paraibana, porém, dispersa em um número reduzido de plantas.

O cajá-manga é bastante apreciado devido às excelentes características organolépticas, sendo rico em fibras, sais minerais (magnésio, potássio, zinco, cobre, cálcio, fósforo e ferro) e algumas vitaminas ( $A$, B1, B6 e C), além de sua importância potencial na agroindústria com diferentes usos (sucos, coquetéis, batidas, licores e sorvetes), porém, sendo mais apreciado pelo consumo fresco (Ishak, Sismail, Noor \& Ahmad 2005; Lorenzi et al., 2006). Há relatos que estes frutos possuem de $35,9 \mathrm{mg} 100 \mathrm{~g}^{-1}$ a $54,2 \mathrm{mg} 100 \mathrm{~g}^{-1}$ de ácido ascórbico, além de carotenoides e compostos fenólicos, ressaltando-se também a presença dos polifenóis extraíveis e da atividade antioxidante (Kohatsu, Zucareli, Brambilla \& Evangelista, 2011). Compostos fitoquímicos possuem propriedades antioxidantes e podem reagir diretamente com espécies químicas reativas, formando produtos pouco reativos, retardando a velocidade da reação de oxidação, através de um ou mais mecanismos relacionados à prevenção de doenças crônicas não transmissíveis, por exemplo, por serem capazes de reduzir o estresse oxidativo (Durazzo, Lucarini, Novellino, Daliu \& Santini, 2019).

Durante o desenvolvimento e amadurecimento dos frutos, surgem uma série de transformações endógenas em consequência de seus metabolismos específicos (Toivonen, 2016). A qualidade de um fruto é evidenciada em determinada fase do desenvolvimento, na qual existe a combinação de diversos atributos, conferindo cor, aroma, sabor, textura característica e, consequentemente, a máxima qualidade comestível. Tais características conferem uma qualidade intrínseca, o que confere aos frutos e aos produtos deles obtidos a qualidade sensorial e nutricional que definem a sua aceitação no mercado (Paliyath \& Murr, 2008).

Devido à escassez de informações sobre aspectos de qualidade e atividade antioxidante de frutas tropicais, nativas e exóticas, que possibilitem agregar valor à sua exploração comercial, faz-se necessário o 
desenvolvendo de estudos que abordem os compostos com propriedades funcionais. Desta forma, este trabalho teve como propósito avaliar as mudanças nos compostos bioativos e na atividade antioxidante, ocorridas durante a maturação de frutos de diferentes genótipos de cajá-mangueira (Spondias cytherea Sonn.).

\section{Material e métodos}

\subsection{Local de desenvolvimento do experimento}

O trabalho foi conduzido no Laboratório de Biologia e Tecnologia de Pós Colheita (LBTPC) do Departamento de Física e Química do Centro de Ciências Agrárias, Campus II da Universidade Federal da Paraíba (DFQ/CCA/UFPB), Areia/Paraíba.

\subsection{Obtenção e seleção dos frutos}

Os frutos foram colhidos pela manhã de cinco genótipos de cajá-mangueira não cultivados no Estado da Paraíba (P1 e P2 localizados no município de Solânea/PB; P3 localizado no município de Cuité de Mamanguape/PB; P4 localizado no município de Campina Grande/PB e P5 localizado no município de João Pessoa/PB), em seguida transportados em caixas térmicas ao laboratório. Essa denominação foi atribuída aos genótipos para facilitar a apresentação dos resultados deste estudo.

No laboratório, os frutos foram lavados e selecionados quanto à ausência de danos físicos e quanto ao estádio de maturação, de acordo com o grau de coloração da casca, sendo classificados em quatro estádios de maturação: TV (totalmente verde); IP (início de pigmentação amarela; AE - (amarelo esverdeado) e PA (predominantemente amarelo) (Figura 1).

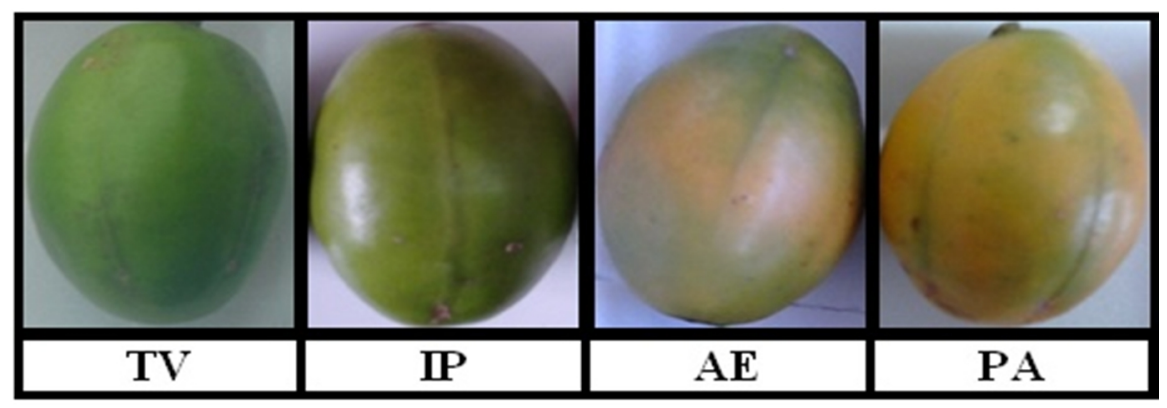

Figura 1. Evolução da maturação de frutos de genótipos de cajá-mangueira (Spondias cytherea Sonn.). TV (totalmente verde), IP (início de pigmentação amarela), AE (amarelo esverdeado) e PA (predominantemente amarelo).

\subsection{Avaliações}

A polpa dos frutos foi obtida a partir do processamento de 15 frutos de cada estádio de maturação e de cada genótipo, sendo avaliadas as seguintes variáveis:

\subsection{Determinação dos compostos bioativos da polpa}

\subsubsection{Carotenoides totais}

A extração e determinação do conteúdo dos carotenoides totais da polpa ( $\mu \mathrm{g} 100 \mathrm{~g}^{-1}$ ) foram baseadas na metodologia descrita por Higby (1962), utilizando $5 \mathrm{~g}$ de polpa para $10 \mathrm{~mL}$ da solução extratora de hexano PA. As leituras foram efetuadas em espectrofotômetro a $450 \mathrm{~nm}$.

\subsubsection{Flavonoides amarelos}


A extração e determinação do conteúdo dos flavonoides amarelos da polpa ( $\left.\mathrm{mg} 100 \mathrm{~g}^{-1}\right)$ foram baseadas na metodologia descrita por Francis (1982), utilizando-se $2 \mathrm{~g}$ de polpa para $10 \mathrm{~mL}$ da solução Etanol PA - HCl $(1,5 \mathrm{~N})(85: 15)$. As leituras foram efetuadas em espectrofotômetro a $374 \mathrm{~nm}$.

\subsubsection{Antocianinas}

A extração e determinação do conteúdo das antocianinas da polpa ( $\left.\mathrm{mg} 100 \mathrm{~g}^{-1}\right)$ foram baseadas na metodologia descrita por Francis (1982), utilizando $2 \mathrm{~g}$ de polpa para $10 \mathrm{~mL}$ da solução Etanol PA - $\mathrm{HCl}$ $(1,5 \mathrm{~N})(85: 15)$. As leituras foram efetuadas em a $535 \mathrm{~nm}$.

\subsection{Atividade antioxidante total (AAT) e polifenóis extraíveis totais (PET)}

\subsubsection{Preparo dos extratos fenólicos}

O extrato fenólico foi determinado seguindo metodologia descrita por Larrauri, Rupérez e SauraCalixto (1997). Tomou-se $3 \mathrm{~g}$ de polpa congelada em freezer a $-8{ }^{\circ} \mathrm{C}$, adicionando $4 \mathrm{~mL}$ de metanol $50 \%$, deixando descansar por 1 hora para extração e centrifugado por 15 minutos em $9.000 \mathrm{rpm}$. Foi retirado o sobrenadante, colocando-o em tubo de ensaio graduado. Adicionou-se $4 \mathrm{~mL}$ de acetona $70 \%$ ao resíduo, deixando-se extrair por 1 hora em repouso, sendo centrifugado por 15 minutos em $9.000 \mathrm{rpm}$. O sobrenadante foi retirado e colocado junto com o primeiro sobrenadante, completando o volume para $10 \mathrm{~mL}$ com água destilada e utilizado para determinar as seguintes análises:

\subsubsection{Determinação polifenóis extraíveis totais (PET)}

O conteúdo de polifenóis extraíveis totais da polpa (mg EAG $100 \mathrm{~g}^{-1}$, EAG-Equivalente de ácido gálico) foi determinado pelo método Folin-Ciocalteu, conforme metodologia descrita por Larrauri et al. (1997). Do extrato produzido no item acima, tomou-se uma alíquota de $200 \mu \mathrm{L}+800 \mu \mathrm{L}$ de água destilada, acrescentado $1 \mathrm{~mL}$ do reagente de Folin Ciocalteu, 2,0 mL de carbonato de sódio $20 \%$ e 2,0 $\mathrm{mL}$ de água destilada. Após 30 minutos, a leitura foi realizada a $700 \mathrm{~nm}$ em espectrofotômetro.

\subsubsection{Determinação da atividade antioxidante total (AAT)}

A Atividade Antioxidante Total da polpa ( $\mu \mathrm{M}$ trolox $\mathrm{g}^{-1}$ de polpa) foi determinada por meio do sistema de co-oxidação ABTS, conforme metodologia descrita por Rufino, Alves, Brito, Pérez-Jiménez, Saura-Calixto, Mancini-Filho (2007). Do extrato fenólico, preparou-se em tubos de ensaio três diluições (10 $\mu \mathrm{L}, 20 \mu \mathrm{L}$ e 30 $\mu \mathrm{L})$, completando para $30 \mu \mathrm{L}$ com água destilada, acrescentando $3 \mathrm{~mL}$ da solução radical ABTS ${ }^{\circ+}\left(2,2^{\prime}\right.$-azinobis (3-etilbenzotiazolin) 6-ácido sulfônico). Após 6 minutos, realizou-se a leitura a $734 \mathrm{~nm} \mathrm{em}$ espectrofotômetro.

\subsubsection{Delineamento experimental e análise estatística}

O delineamento experimental foi o inteiramente casualizado, em esquema fatorial $5 \times 4$, sendo cinco genótipos e quatro estádios de maturação, em seis repetições. Os dados foram submetidos à análise de variância (ANOVA), utilizando o programa estatístico SISVAR versão 5.1 (Ferreira, 2014). As médias foram comparadas pelo teste de Tukey a $5 \%$ de probabilidade de erro $(p \leq 0,05)$. Para a análise multivariada, utilizouse a análise de componentes principais, tendo como base a resposta aos fatores estudados para o autovalor da matriz de correlação $(\lambda)$. Já para a determinação da contribuição dos componentes bioativos com a atividade antioxidante da polpa dos frutos dos diferentes genótipos, foi realizada a correlação de Pearson. Foi usado o programa JMP® (SAS Institute).

\section{Resultados}

\subsection{Compostos bioativos}


Os frutos de cajá-mangueira tiveram origem de diferentes localidades e, embora sendo avaliados nos mesmos estádios de maturação com base na coloração da cassa, os conteúdos dos compostos variaram tanto com o avanço da maturação quanto entre os diferentes genótipos. Para o conteúdo de carotenoides totais da polpa, houve diferenças $(p \leq 0,05)$ entre os estádios de maturação para os genótipos P2, P3, P4 e P5. Independentemente da origem do fruto, o aumento é resultado do processo de maturação do fruto. $O$ maior incremento no conteúdo de carotenoides totais da polpa foi observado nos frutos do genótipo P3, que apresentou $80,10 \mu \mathrm{g} 100 \mathrm{~g}^{-1}$ no estádio TV, atingindo 263,75 $\mu \mathrm{g} 100 \mathrm{~g}^{-1}$ quando os frutos apresentavam coloração predominantemente amarela (estádio PA) (Tabela 1).

Tabela 1. Conteúdo de Carotenoides Totais da polpa de frutos de cajá-mangueira, colhidos em diferentes estádios de maturação.

\begin{tabular}{ccccc}
\hline \multirow{2}{*}{ Genótipo } & \multicolumn{4}{c}{ Estádios de Maturação } \\
\cline { 2 - 5 } & TV & $\mathrm{IP}$ & $\mathrm{AE}$ & $\mathrm{PA}$ \\
\hline & & $\mu \mathrm{g} 100 \mathrm{~g}^{-1}$ & \\
P1 & $126,01 \mathrm{Aa}$ & $122,31 \mathrm{Aab}$ & $135,58 \mathrm{Aa}$ & $119,98 \mathrm{Ab}$ \\
P2 & $102,41 \mathrm{Cab}$ & $89,92 \mathrm{Cb}$ & $144,51 \mathrm{Ba}$ & $202,84 \mathrm{Ab}$ \\
P3 & $80,10 \mathrm{Bc}$ & $140,63 \mathrm{Ba}$ & $117,01 \mathrm{Ba}$ & $263,75 \mathrm{Aa}$ \\
P4 & $39,10 \mathrm{Bd}$ & $33,60 \mathrm{Bc}$ & $28,78 \mathrm{Bb}$ & $53,30 \mathrm{Ad}$ \\
P5 & $45,54 \mathrm{BCd}$ & $50,46 \mathrm{Bc}$ & $35,15 \mathrm{Cb}$ & $81,88 \mathrm{Abc}$ \\
\hline Média Geral & 78,63 & 87,39 & 92,21 & 144,35 \\
\hline
\end{tabular}

TV - Totalmente Verde; IP - Início de Pigmentação Amarela; AE - Amarelo Esverdeado e PA - Predominantemente Amarelo). *Médias seguidas de letras iguais, maiúsculas na linha e minúsculas na coluna, não diferem entre si pelo teste de Tukey $(P \leq 0,05)$.

Para o conteúdo de flavonoides amarelos, observou-se redução com o avanço da maturação partindo de 2,38 mg $100 \mathrm{~g} \mathrm{~g}^{-1}$ no estádio TV para 1,93 mg $100 \mathrm{~g}^{-1}$ no estádio PA (Tabela 2). O genótipo P3

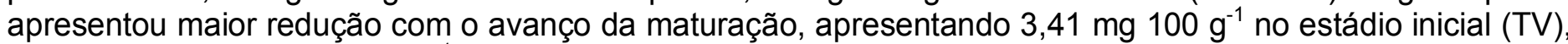

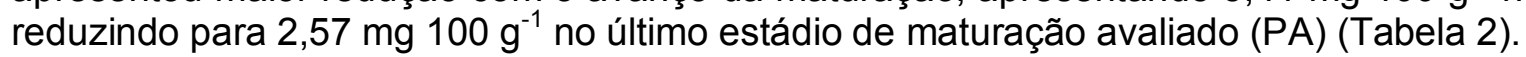

Houve diferença $(p \leq 0,05)$ entre os estádios de maturação quanto ao conteúdo de flavonoides amarelos da polpa dos frutos dos genótipos P2, P3 e P5 (Tabela 2). Os frutos do genótipo P2 apresentaram os maiores conteúdos nos estádios TV, IP e AE, com valores médios de 2,30, 2,32 e 2,13 mg $100 \mathrm{~g}^{-1}$, respectivamente. Para os frutos do genótipo $\mathrm{P} 3$, os maiores conteúdos foram expressos no estádio $A E$, com média de 3,98 mg $100 \mathrm{~g}^{-1}$. Já para os frutos do genótipo P5, os maiores conteúdos foram observados nos estádios TV e IP (2,28 e 2,14 mg $100 \mathrm{~g}^{-1}$, respectivamente), sendo iguais entre si e diferindo dos demais estádios de maturação (Tabela 2).

Desse modo, os maiores conteúdos de flavonoides amarelos foram expressos nos três primeiros estádios de maturação (TV, IP e AE), nos quais as médias variaram de 1,33 a 3,98 mg $100 \mathrm{~g}^{-1}$. Diferenças foram observadas entre os genótipos para todos os estádios de maturação avaliados (Tabela 2), no qual o conteúdo dos frutos do genótipo P3 foi superior e diferiu dos demais genótipos, com conteúdo médio de 3,41 mg $100 \mathrm{~g}^{-1}$ (TV), 3,54 mg $100 \mathrm{~g}^{-1}$ (IP), 3,98 mg $100 \mathrm{~g}^{-1}$ (AE) e 2,57 mg $100 \mathrm{~g}^{-1}$ (PA) (Tabela 2).

Tabela 2. Conteúdo de flavonoides amarelos da polpa de frutos da cajá-mangueira, colhidos em diferentes estádios de maturação.

\begin{tabular}{ccccc}
\hline Genótipo & \multicolumn{4}{c}{ Estádios de Maturação } \\
\cline { 2 - 5 } & TV & IP & AE & PA \\
P1 & $1,51 \mathrm{Ac}$ & $1,33 \mathrm{Ac}$ & $1,35 \mathrm{Ac}$ & $1,29 \mathrm{Ac}$ \\
P2 & $2,30 \mathrm{Ab}$ & $2,32 \mathrm{Ab}$ & $2,13 \mathrm{Ab}$ & $1,78 \mathrm{Bb}$ \\
P3 & $3,41 \mathrm{Ba}$ & $3,54 \mathrm{Ba}$ & $3,98 \mathrm{Aa}$ & $2,57 \mathrm{Ca}$ \\
P4 & $2,39 \mathrm{Ab}$ & $2,18 \mathrm{Ab}$ & $2,01 \mathrm{Ab}$ & $2,09 \mathrm{Ab}$ \\
P5 & $2,28 \mathrm{Ab}$ & $2,14 \mathrm{ABb}$ & $1,81 \mathrm{Bb}$ & $1,90 \mathrm{Bb}$ \\
\hline Média Geral & 2,38 & 2,30 & 2,26 & 1,93 \\
\hline
\end{tabular}

TV - Totalmente Verde; IP - Início de Pigmentação Amarela; AE - Amarelo Esverdeado e PA - Predominantemente Amarelo). *Médias seguidas de letras iguais, maiúsculas na linha e minúsculas na coluna, não diferem entre si pelo teste de $T u k e y(P \leq 0,05)$. 
Para o conteúdo médio de antocianinas da polpa dos frutos de cajá-mangueira, observou-se que ocorreu aumento com o avanço da maturação nos genótipos P1 e P4, partindo de 0,23 e 0,29 mg $100 \mathrm{~g}^{-1}$ no estádio TV (totalmente verde) para 0,45 e $0,53 \mathrm{mg} 100 \mathrm{~g}^{-1}$ no estádio PA (totalmente amarelo), respectivamente (Tabela 3 ).

Tabela 3. Conteúdo médio de antocianinas da polpa de frutos da cajá-mangueira, colhidos em diferentes estádios de maturação.

\begin{tabular}{ccccc}
\hline Genótipo & \multicolumn{4}{c}{ Estádios de Maturação } \\
\cline { 2 - 5 } & TV & $\mathrm{IP}$ & $\mathrm{AE}$ \\
\hline P1 & $0,23 \mathrm{Bd}$ & $0,29 \mathrm{Bc}$ & $0,28 \mathrm{Bb}$ & $0,45 \mathrm{Aab}$ \\
P2 & $0,38 \mathrm{Ab}$ & $0,39 \mathrm{Aabc}$ & $0,39 \mathrm{Aab}$ & $0,36 \mathrm{Ab}$ \\
P3 & $0,36 \mathrm{Bbc}$ & $0,46 \mathrm{Aa}$ & $0,48 \mathrm{Aa}$ & $0,38 \mathrm{Bb}$ \\
P4 & $0,29 \mathrm{Bcd}$ & $0,32 \mathrm{Bbc}$ & $0,53 \mathrm{Aa}$ & $0,53 \mathrm{Aa}$ \\
P5 & $0,53 \mathrm{Aa}$ & $0,42 \mathrm{ABab}$ & $0,44 \mathrm{ABa}$ & $0,41 \mathrm{Bb}$ \\
\hline Média Geral & 0,36 & 0,38 & 0,43 & 0,42
\end{tabular}

TV - Totalmente Verde; IP - Início de Pigmentação Amarela; AE - Amarelo Esverdeado e PA - Predominantemente Amarelo). *Médias seguidas de letras iguais, maiúsculas na linha e minúsculas na coluna, não diferem entre si pelo teste de Tukey $(P \leq 0,05)$.

Foi observado que o conteúdo de polifenóis extraíveis totais (PET) da polpa de frutos de cajámangueira variou entre 54,17 e $64,48 \mathrm{mg} 100 \mathrm{~g}^{-1}$ entre os genótipos para todos os estádios de maturação avaliados (Tabela 4). As maiores médias encontradas no estádio de maturação TV foram para os genótipos P1 e P5 (68,44 e 64,75 mg $\left.100 \mathrm{~g}^{-1}\right)$, respectivamente, sendo iguais entre si e diferindo dos demais genótipos.

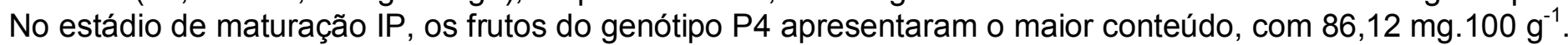
Enquanto, no estádio PA os frutos dos genótipos P4 e P3 aprestaram os maiores conteúdos $(78,05,74,49$ $\left.\mathrm{mg} .100 \mathrm{~g}^{-1}\right)$, respectivamente. Os maiores conteúdos de PET foram obtidos na polpa no estádio $A E$, nos frutos do genótipo P4, com média de $79,80 \mathrm{mg} \cdot 100 \mathrm{~g}^{-1}$ (Tabela 4).

Tabela 4. Conteúdo de Polifenóis Extraíveis Totais - PET de frutos da cajá-mangueira, colhidos em diferentes estádios de maturação.

\begin{tabular}{ccccc}
\hline Genótipo & \multicolumn{4}{c}{ Estádios de Maturação } \\
\cline { 2 - 5 } & TV & IP & AE & PA \\
\hline P1 & mg EAG $100 \mathrm{~g}^{-1}$ & $63,49 \mathrm{Ab}$ & $54,12 \mathrm{Bb}$ \\
P2 & $68,44 \mathrm{Aa}$ & $66,79 \mathrm{Ab}$ & $57,62 \mathrm{Ab}$ & $54,45 \mathrm{Ab}$ \\
P3 & $56,35 \mathrm{Acd}$ & $55,26 \mathrm{Acd}$ & $43,89 \mathrm{Db}$ & $74,49 \mathrm{Aa}$ \\
P4 & $55,77 \mathrm{Bbc}$ & $49,96 \mathrm{Cd}$ & $79,80 \mathrm{Aa}$ & $78,05 \mathrm{Aa}$ \\
P5 & $64,54 \mathrm{Bd}$ & $86,12 \mathrm{Aa}$ & $49,92 \mathrm{Bb}$ & $53,46 \mathrm{Bb}$ \\
\hline Média Geral & 54,17 & $64,29 \mathrm{Abc}$ & 58,94 & 62,91 \\
\hline
\end{tabular}

TV - Totalmente Verde; IP - Início de Pigmentação Amarela; AE - Amarelo Esverdeado e PA - Predominantemente Amarelo). *Médias seguidas de letras iguais, maiúsculas na linha e minúsculas na coluna, não diferem entre si pelo teste de $T u k e y(P \leq 0,05)$.

\subsection{Atividade antioxidante total (AAT)}

A atividade antioxidante (AAT) da polpa dos frutos de cajá-mangueira oscilou com o avanço da maturação, aumentando em média de $5,81 \mu \mathrm{M}$ trolox $\mathrm{g}^{-1}$ de polpa (estádio TV) para $6,13 \mu \mathrm{M}$ trolox $\mathrm{g}^{-1}$ de polpa (estádio IP), diminuindo, em seguida, para $5,24 \mu \mathrm{M}$ trolox $\mathrm{g}^{-1}$ de polpa (estádio $\mathrm{AE}$ ), com posterior aumento para 6,69 $\mu \mathrm{M}$.trolox $\mathrm{g}^{-1}$ de polpa (estádio PA) (Tabela 5).

A AAT diferiu $(p \leq 0,05)$ entre os estádios de maturação para os genótipos $P 1, P 3, P 4$ e P5. Para o genótipo P1, a maior média de AAT encontrada foi para o estádio de maturação IP $\left(6,66 \mu \mathrm{M}\right.$ trolox $\left.\mathrm{g}^{-1}\right)$, sendo igual aos estádios TV e PA. Em P3, a maior AAT foi a de frutos do estádio PA $\left(11,04 \mu \mathrm{M}\right.$ trolox $\left.\mathrm{g}^{-1}\right)$; enquanto para o P4, foi para o IP $\left(6,47 \mu \mathrm{M}\right.$ trolox $\mathrm{g}^{-1}$ de polpa) e $\mathrm{AE}\left(6,16 \mu \mathrm{M}\right.$ trolox $\mathrm{g}^{-1}$ de polpa). Para o genótipo P5, os maiores valores foram para os estádios de maturação TV e $A E\left(8,97\right.$ e $6,98 \mu \mathrm{M}$ trolox $\mathrm{g}^{-1}$ de polpa), respectivamente, sendo iguais entre si e diferindo dos demais estádios de maturação (Tabela 5). 
Observou-se também diferença $(p \leq 0,05)$ para a AAT entre os genótipos em todos os estádios de maturação avaliados. Os genótipos $P 1$ e $P \overline{5}$ apresentaram os maiores valores de AAT, no estádio TV, com 6,11 e $8,97 \mu \mathrm{M}$ trolox $\mathrm{g}^{-1}$, respectivamente. No estádio IP, o genótipo P1 apresentou a maior AAT com média de $6,66 \mu \mathrm{M}$ trolox $\mathrm{g}^{-1}$. Para o estádio AE, os genótipos P1, P2, P4 e P5 foram os com AAT mais elevada, com $5,60,4,86,6,16$ e 5,79 $\mu \mathrm{M}$ trolox $\mathrm{g}^{-1}$, respectivamente. Para o estádio PA, a AAT de frutos do genótipo P1 foi superior com 11,04 $\mu \mathrm{M}$ trolox $\mathrm{g}^{-1}$ (Tabela 5 ).

Tabela 5. Atividade antioxidante total (AAT) de frutos da cajá-mangueira, colhidos em diferentes estádios de maturação.

\begin{tabular}{ccccc}
\hline \multirow{2}{*}{ Genótipo } & \multicolumn{4}{c}{ Estádios de Maturação } \\
\cline { 2 - 5 } & TV & IP & AE & PA \\
\hline P1 & $6,11 \mathrm{ABab}$ & $6,66 \mathrm{AM}$ trolox g & & $5,89 \mathrm{ABb}$ \\
P2 & $5,44 \mathrm{Ab}$ & $6,10 \mathrm{Aa}$ & $5,60 \mathrm{Bab}$ & $5,06 \mathrm{Ab}$ \\
P3 & $5,18 \mathrm{Bb}$ & $4,43 \mathrm{Bb}$ & $3,81 \mathrm{Bc}$ & $11,04 \mathrm{Aa}$ \\
P4 & $3,36 \mathrm{Cb}$ & $6,47 \mathrm{Aa}$ & $6,16 \mathrm{Aa}$ & $5,46 \mathrm{Bb}$ \\
P5 & $8,97 \mathrm{Aa}$ & $6,98 \mathrm{ABa}$ & $5,79 \mathrm{Ba}$ & $5,98 \mathrm{Bb}$ \\
\hline Média Geral & 5,81 & 6,13 & 5,24 & 6,69 \\
\hline
\end{tabular}

TV - Totalmente Verde; IP - Início de Pigmentação Amarela; AE - Amarelo Esverdeado e PA - Predominantemente Amarelo). ${ }^{*}$ Médias seguidas de letras iguais, maiúsculas na linha e minúsculas na coluna, não diferem entre si pelo teste de Tukey $(P \leq 0,05)$.

\subsection{Análise multivariada}

Avaliando os autovetores na Tabela 6, verifica-se que todos os compostos bioativos e a atividade antioxidante avaliados nos frutos de cajá-mangueira são importantes para explicar a variabilidade ocorrida durante a maturação dos frutos, sendo os carotenoides totais, flavonoides e polifenóis extraíveis totais relacionados ao componente principal 1 , respondendo por $67,8 \%$ da variabilidade. Enquanto as antocianinas juntamente com a atividade antioxidante respondem por $20,1 \%$ da variabilidade no componente principal 2 . Dessa forma, dois componentes explicam satisfatoriamente a variabilidade entre os estádios de maturação de cajá-manga, com 84,8 \% de variância acumulada independentemente do genótipo.

Tabela 6. Autovetores para os dois componentes principais (CP1 e CP2) com base na correlação de variáveis analisadas em frutos de cajá-manga em 4 estádios de maturação.

\begin{tabular}{ccc}
\hline Variáveis & CP1 & CP2 \\
\hline Carotenoides totais & $0,53^{*}$ & $-0,04$ \\
Flavonoides amarelos & $-0,53^{*}$ & 0,09 \\
Antocianinas & 0,37 & $-0,72^{*}$ \\
Polifenóis extraíveis totais & $0,38^{*}$ & 0,20 \\
Atividade antioxidante total & 0,40 & $0,65^{*}$ \\
\hline Autovalores & 3,39 & 1,01 \\
Variância acumulada & 67,8 & 20,1 \\
\hline
\end{tabular}

*Variáveis significativas para o componente.

Observa-se na Figura 2 que os carotenoides, as antocianinas e atividade antioxidante total (ABTS ${ }^{\bullet+}$ ) da polpa de cajá-manga apresentaram-se mais intensos quando a maturação encontrava-se mais avançada (estádio PA), sendo a atividade antioxidante principalmente correlacionada com o estádio PA. Enquanto que, nos três estádios iniciais (TV, IP e AE), compondo outro grupo, os flavonoides apresentaram maior expressão (coloração mais intensa) e a atividade antioxidante total foi reduzida, comparativamente aos estádios mais avançados, estando os polifenóis extraíveis totais mais elevados no estádio de maturação IP. Assim, nota-se que a atividade antioxidante total em cajá-manga, determinada pela captura do radical $A B T S^{{ }^{++}}$, foi expressa principalmente pelos conteúdos de carotenoides, antocianinas e polifenóis extraíveis totais da polpa quando os frutos se encontram mais maduros. 


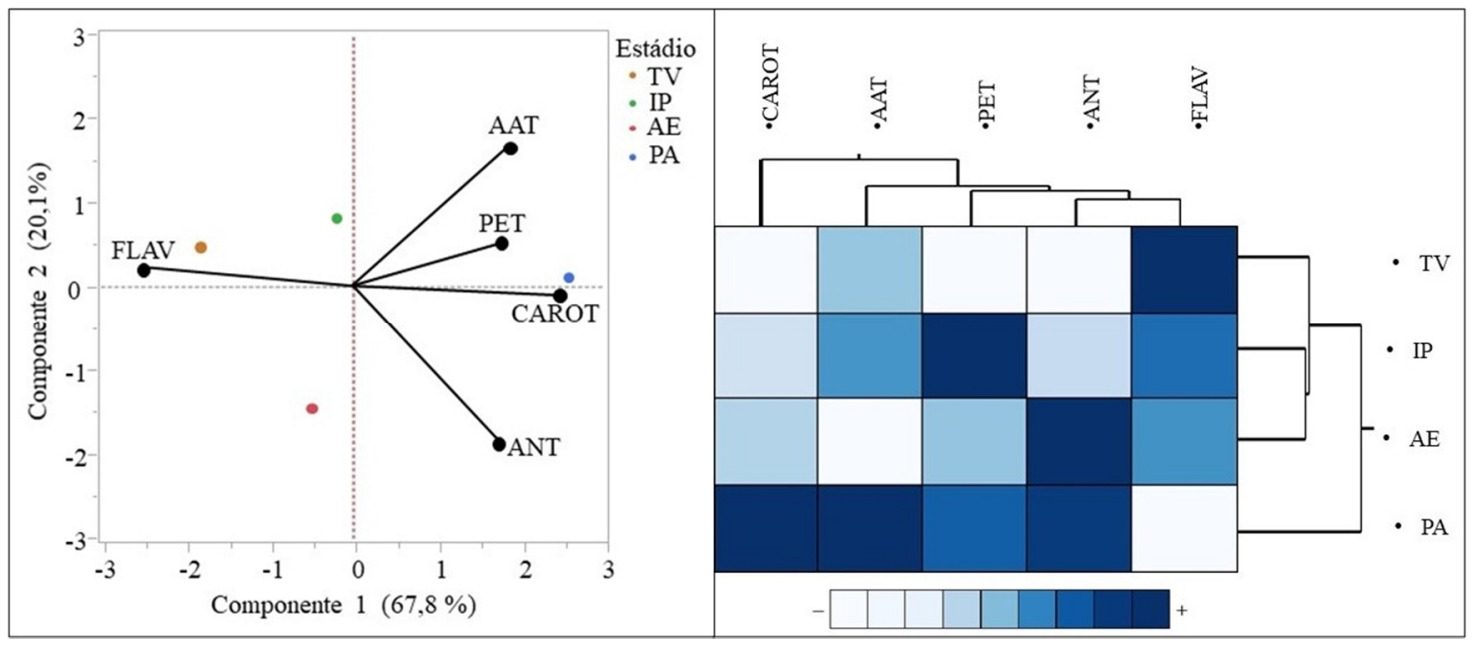

Figura 2. Análise de componentes principais e agrupamento (Two-way clustering) para variáveis analisadas em frutos de cajá-manga em quatro estádios de maturação com base no critério cúbico para agrupamento.

\section{Discussão}

\subsection{Compostos bioativos}

O conteúdo de carotenoides totais da polpa dos frutos de cajá-mangueira avaliados neste trabalho aumentou com o avanço da maturação. De acordo com Rodriguez-Amaya (2008), a variação no conteúdo de carotenoides pode estar relacionada a fatores edafoclimáticos (tipo de solo, condições de cultivo, condições climáticas e exposição à luz solar) e/ou genéticos (espécies vegetais e estádio de maturação). Além destes, pode-se destacar também as condições de processamento e armazenamento dos frutos após a colheita.

Os valores descritos neste trabalho para carotenoides totais da polpa de cajá-manga estão próximos aos relatados por Barreto, Benassib e Mercadante (2009) que, estudando 18 frutíferas tropicais obtidas no Brasil, dentre elas o cajá-mangueira, reportaram valor de 1,2 mg $100 \mathrm{~g}^{-1}$. Melo e Andrade (2010), avaliando frutos de umbuzeiro nos estádios de maturação semimaduro e maduro, observaram o aumento dos carotenoides com o avanço da maturação, porém com resultados superiores aos relatados neste trabalho, visto que os frutos semimaduros apresentaram média de $170,0 \mu \mathrm{g} 100 \mathrm{~g} \mathrm{~g}^{-1}$ e os frutos maduros de $302,0 \mu \mathrm{g}$ $100 \mathrm{~g}^{-1}$. Yahia e Ornelas-Paz (2010) citam que os carotenoides apresentam um papel importante na saúde e nutrição humana, podendo minimizar o risco de câncer e doenças coronárias, devido à atividade da provitamina A.

O conteúdo de flavonoides amarelos reduziu com o avanço da maturação, partindo de 2,38 mg 100 $\mathrm{g}^{-1}$ no estádio TV para $1,93 \mathrm{mg} 100 \mathrm{~g}^{-1}$ no estádio PA. Os maiores conteúdos foram expressos nos três primeiros estádios de maturação (TV, IP e AE), no qual as médias variaram de 1,33 a 3,98 $\mathrm{mg}^{100 \mathrm{~g}^{-1}}$ (Tabela 2). Estes valores são inferiores aos relatados por Rufino et al. (2010) para frutos de outras Spondias (cajazeira e umbuzeiro) com 7,1 e 6,9 mg.100 g ${ }^{-1}$, respectivamente.

Os maiores conteúdos de antocianinas foram evidenciados entre os estádios de maturação IP e PA. No entanto, no estádio TA, os genótipos P3, P4 e P5 mostraram os maiores valores com 0,48, 0,53 e 0,44 mg $100 \mathrm{~g}^{-1}$, respectivamente (Tabela 3). Estes valores corroboram com os relatados por Rufino et al. (2010), avaliando dezoito espécies de frutos não tradicionais, oriundos de diferentes estados do Brasil, em que frutos de umbuzeiro (Spondias tuberosa Arr. Cam.) apresentaram conteúdo médio de antocianinas de 0,30 mg 100 $\mathrm{g}^{-1}$ de polpa.

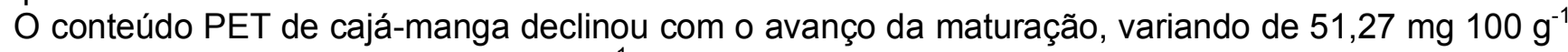
no estádio de maturação TA a $64,48 \mathrm{mg} 100 \mathrm{~g} \mathrm{~g}^{-1}$ no estádio IP (Tabela 4). Esta redução no conteúdo de PET com o avanço da maturação também foi reportada por Ishak et al. (2005) que, ao avaliarem as mudanças nas características físicas e químicas em três estádios de maturação de frutos de cajá-mangueira, observaram que o conteúdo de PET reduziu de $686,50 \mathrm{mg} 100 \mathrm{~g}^{-1}$ no estádio verde para 338,00 mg $100 \mathrm{~g}^{-1}$ no maduro. Este comportamento também foi relatado por Silva et al. (2013) que, ao avaliarem os compostos bioativos e atividade antioxidante de frutos de cajazeira (Spondias mombin L.) colhidos em três estágios de maturação, 


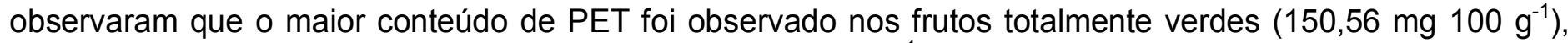

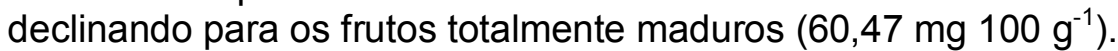

Os valores de PET encontrados neste trabalho foram superiores aos reportados por Lim, Lim e Tee (2007) que reportaram valores de $33,00 \mathrm{mg} 100 \mathrm{~g} \mathrm{~g}^{-1} \mathrm{em}$ frutos de cajá-mangueira. No entanto, foi inferior aos resultados obtidos por Ishak et al. (2005) e Barreto et al. (2009) que observaram valores médios de 338,00 e $99,4 \mathrm{mg} 100 \mathrm{~g}^{-1}$ de PET na mesma espécie e com frutos maduros, respectivamente.

Comparando o conteúdo de PET dos frutos de cajá-mangueira avaliados neste trabalho com os de frutos de espécies do mesmo gênero botânico (Spondias), o teor de polifenóis foi semelhante ao citado por Gondim (2012) e Silva et al. (2013) que reportaram conteúdo médio de PET de 44,68 e 60,47 mg $100 \mathrm{~g}^{-1}$, para frutos de maduros de umbu-cajazeira (Spondias sp.) e cajazeira (Spondias mombin L.), respectivamente. No entanto, inferior aos citados para frutos de cajá maduros (Spondias mombin L.): $249,00 \mathrm{mg}^{100 \mathrm{~g}^{-1}}$ (Vasco, Ruales \& Kamal-Eldin, 2008), 72,00 mg $100 \mathrm{~g}^{-1}$ (Rufino et al., 2010), 260,21 mg $100 \mathrm{~g}^{-1}$ (Tiburski, Rosenthal, Deliza, Godoy \& Pacheco, 2011) e 925,84 mg $100 \mathrm{~g}^{-1}$ (Silva et al., 2014).

Em polpa de cajá colhidos no estádio de maturação comercial (coloração da casca totalmente

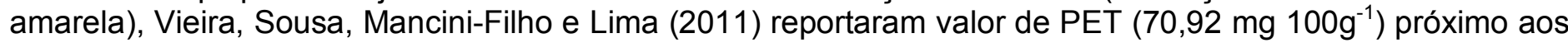
reportados neste trabalho. Esta variação do conteúdo de PET ao longo da maturação dos frutos está, provavelmente, ligada as condições edafoclimáticas e do estádio de maturação dos frutos. As condições sob as quais as plantas são mantidas influenciam significativamente nos conteúdos desses compostos, podendo haver diferenças no perfil de compostos fenólicos tanto quantitativa quanto qualitativamente (Sousa, Mattietto, Chisté, \& Carvalho, 2018).

\subsection{Atividade antioxidante total (AAT)}

A atividade antioxidante (AAT) da polpa dos frutos de cajá-mangueira avaliados neste trabalho oscilou com o avanço da maturação, apresentado um aumento de $13 \%$ dos frutos no estádio TV para o estádio PA. Este comportamento de oscilação e aumento da atividade antioxidante também foi observado por Wang, Chen e Wang (2009) que, ao avaliarem a influência da luz e da maturidade na qualidade de framboesas vermelhas, concluíram que AAT foi superior em frutos totalmente maduros.

Barreto et al. (2009) apresentaram atividade antioxidante em cajá-manga de 21,0 $\mu \mathrm{M}_{\text {trolox g }}{ }^{-1}$, enquanto, Vieira et al. (2011) reportaram para frutos da cajazeira $0,140 \mu \mathrm{M}$ trolox $\mathrm{g}^{-1}$ de polpa, inferiores ao observado neste trabalho para cajá-manga. Rufino et al. (2010) encontraram para cajazeira e umbuzeiro valores médios de 40,7 e 77,0 $\mu \mathrm{M}$ trolox $\mathrm{g}^{-1}$ de polpa, respectivamente, superiores aos observados neste trabalho para o cajá-manga.

A AAT da polpa de cajá-manga apresentada neste trabalho, está próxima ao de frutos tradicionais de valor econômico no mercado, a exemplo da polpa de goiaba, uva, amora, açaí e graviola, que apresentaram valores médios de 5,9,7,0,4,3,6,9, 2,9 e $\mu \mathrm{M}$ trolox $\mathrm{g}^{-1}$, respectivamente, e superior a polpa de abacaxi e maracujá, com 0,5 e 0,9 $\mu \mathrm{M}$ trolox g ${ }^{-1}$, respectivamente (Kuskoski, Asuero, Morales \& Fett, 2006).

De acordo com Leong e Shui (2002), as frutas apresentam em sua constituição química grande variedade de compostos bioativos que podem possuir ação antioxidante. Dentre estes compostos bioativos, destacam-se os polifenóis e os carotenoides. Tendo como base os resultados obtidos neste estudo, verificouse que o conteúdo destes constituintes variou quanto ao genótipo e ao estádio de maturação dos frutos, podendo ter contribuído para maior atividade antioxidante dos genótipos avaliados.

\subsection{Análise multivariada}

A correlação dos carotenoides, antocianinas e atividade antioxidante total $\left(\mathrm{ABTS}^{\bullet+}\right)$ da polpa de cajámanga em relação ao avanço da maturação, também foi encontrada por Gondim (2012) e Silva, Silva, Silva, Mendonça, Alves e Dantas (2012), que reportaram correlação entre os conteúdos de carotenoides e polifenóis extraíveis totais, com a capacidade antioxidante pelo método ABTS, em frutos de umbuzeiro (Spondias tuberosa Arr. Cam.) e cajazeira (Spondias mombin L.), respectivamente. Estes dados corroboram com Dudonné, Vitrac, Coutiere, Woillez eMérilon (2009), visto que estes autores afirmaram que a presença de compostos fenólicos em extratos de vegetais apresenta alta correlação positiva com a capacidade antioxidante.

\section{Conclusões}


Os frutos de cajá mangueira nos estádios mais avançados de maturação acumularam grupos de compostos considerados funcionais, como carotenoides e compostos fenólicos.

O genótipo P3, localizado no município de Cuité de Mamanguape/PB, destacou-se dentro os demais por apresentar frutos com maior conteúdo de carotenoides totais e flavonoides amarelos ao final da maturação (estádio predominantemente amarelo - PA), enquanto, os frutos dos genótipos P1 e P5 apresentaram maior capacidade antioxidante.

\section{Agradecimentos}

Ao Conselho Nacional de Desenvolvimento Científico e Tecnológico (CNPq) pelo auxílio financeiro na execução do presente trabalho e pela concessão da bolsa de iniciação cientifica ao primeiro autor.

\section{Referências}

Almeida Melo, E., \& Sena Andrade, R.A.M. (2010). Compostos bioativos e potencial antioxidante de frutos do umbuzeiro. Brazilian Journal of Food \& Nutrition/Alimentos e Nutrição, 21(3). http://servbib.fcfar.unesp.br/seer/index.php/alimentos/article/view/1208

Barreto, G.P., Benassi, M.T., \& Mercadante, A.Z. (2009). Bioactive compounds from several tropical fruits and correlation by multivariate analysis to free radical scavenger activity. Journal of the Brazilian Chemical Society, 20(10), 1856-1861. http://dx.doi.org/10.1590/S0103-50532009001000013

Dantas, A.L., Silva, S.D.M., Dantas, R.L., Sousa, A.S.B.D., \& Schunemann, A.P.P. (2016). Desenvolvimento, fisiologia da maturação e indicadores do ponto de colheita de frutos da umbugueleira (Spondias sp.). Revista Brasileira de Fruticultura, 38(1), 33-42. http://dx.doi.org/10.1590/0100-2945-271/14

Dudonne, S., Vitrac, X., Coutiere, P., Woillez, M., \& Mérillon, J.M. (2009). Comparative study of antioxidant properties and total phenolic content of 30 plant extracts of industrial interest using DPPH, ABTS, FRAP, SOD, and ORAC assays. Journal of agricultural and food chemistry, 57(5), 1768-1774. http://dx.doi.org/10.1021/jf803011r

Durazzo, A., Lucarini, M., Novellino, E., Daliu, P., \& Santini, A. (2019). Fruit-based juices: Focus on antioxidant properties-Study approach and update. Phytotherapy Research, 33(7), 1754-1769. http://dx.doi.org/10.1002/ptr.6380

Ferreira, D.F. (2014). Sisvar: A Guide for its Bootstrap procedures in multiple comparisons. Ciência e agrotecnologia, 38(2), 109-112. http://dx.doi.org/10.1590/S1413-70542014000200001

Fonseca, N., Machado, C.D.F., Silva Junior, J.F., Carvalho, R.D.S., Ritzinger, R., Alves, R., \& Maia, M. (2017). Umbu: cajá e espécies afins: Spondias spp. Embrapa Mandioca e Fruticultura-Fôlder/Folheto/Cartilha (INFOTECA-E).

Francis, F.J. (1982). Analysis of anthocyanins. In: Markaki, P. Anthocyanins as food colors. Nova York: Academic Press, Inc.

Gondim, P.J.S. (2012). Identificação de carotenoides e quantificação de compostos bioativos e atividade antioxidante em frutos do gênero Spondias. Tese de Doutorado - Universidade Federal da Paraíba, Areia, PB, Brasil.

Higby, W.K. (1962). A simplified method for determination of some aspects of the carotenoid distribution in natural and carotene-fortified orange juice. journal of food science, 27(1), 42-49. https://doi.org/10.1111/i.13652621.1962.tb00055.x

Ishak, S.A., Ismail, N., Noor, M.A.M., \& Ahmad, H. (2005). Some physical and chemical properties of ambarella (Spondias cytherea Sonn.) at three different stages of maturity. Journal of Food Composition and Analysis, 18(8), 819-827. http://dx.doi.org/10.1016/i.jfca.2004.11.007

Kohatsu, D.S., Zucareli, V., Brambilla, W.P., \& Evangelista, R.M. (2011). Qualidade de frutos de cajá-manga armazenados sob diferentes temperaturas. Revista Brasileira de Fruticultura, 33(SPE1), 344-349. http://dx.doi.org/10.1590/S0100-29452011000500043 
Koubala, B.B., Kansci, G., \& Ralet, M.C. (2018). Ambarella- Spondias cytherea. Exotic Fruits, 1522. https://doi.org/10.1016/b978-0-12-803138-4.00005-8

Kuskoski, E.M., García Asuero, A., Morales Millán, M.T., \& Fett, R. (2006). Frutos tropicais silvestres e polpas de frutas congeladas: atividade antioxidante, polifenóis e antocianinas. Ciência Rural, 36 (4), 1283-1287. http://dx.doi.org/10.1590/S0103-84782006000400037

Larrauri, J.A., Rupérez, P., \& Saura-Calixto, F. (1997). Effect of drying temperature on the stability of polyphenols and antioxidant activity of red grape pomace peels. Journal of agricultural and food chemistry, 45(4), 1390-1393. http://dx.doi.org/10.1021/if960282f

Leong, L.P., \& Shui, G. (2002). An investigation of antioxidant capacity of fruits in Singapore markets. Food chemistry, 76(1), 69-75. https://doi.org/10.1016/S0308-8146(01)00251-5

Lim, Y.Y., Lim, T.T., \& Tee, J.J. (2007). Antioxidant properties of several tropical fruits: A comparative study. Food chemistry, 103(3), 1003-1008. http://dx.doi.org/10.1016/i.foodchem.2006.08.038

Lorenzi, H., Bacher, L., Lacerda, M., \& Sartori, S. (2006). Frutas brasileiras e exóticas cultivadas (de consumo in natura). São Paulo: Instituto plantarum de estudos da Flora, 640.

Maria do Socorro, M.R., Alves, R.E., Brito, E.S., Pérez-Jiménez, J., Saura-Calixto, F., \& Mancini-Filho, J. (2010). Bioactive compounds and antioxidant capacities of 18 non-traditional tropical fruits from Brazil. Food chemistry, 121(4), 996-1002. https://doi.org/10.1016/j.foodchem.2010.01.037

Neves, L.C., Tosin, J.M., Benedette, R.M., \& Cisneros-Zevallos, L. (2015). Post-harvest nutraceutical behaviour during ripening and senescence of 8 highly perishable fruit species from the Northern Brazilian Amazon region. Food Chemistry, 174, 188-196. http://dx.doi.org/10.1016/i.foodchem.2014.10.111

Paliyath, G., \& Murr, D.P. (2006). Biochemistry of fruits. In: Huy, Y. H. Food biochemistry and food processing. Blackwell Publishing, Oxford, 487-514.

Rodriguez-Amaya, D.B. (2000). Some considerations in generating carotenoid data for food composition tables. Journal of food composition and analysis, 13(4), 641-647. https://doi.org/10.1006/ifca.2000.0915

Rufino, M.D.S.M., Alves, R.E., Brito, E.S., Morais, S.M., Sampaio, C.D.G., Pérez-Jimenez, J., \& Saura-Calixto, F.D. (2007). Metodologia científica: determinação da atividade antioxidante total em frutas pela captura do radical livre DPPH. Embrapa Agroindústria Tropical-Comunicado Técnico (INFOTECA-E). https://www.infoteca.cnptia.embrapa.br/bitstream/doc/426953/1/Cot127.pdf

Silva, F.V.G.D., Silva, S.D.M., Silva, G.C.D., Mendonça, R.M.N., Alves, R.E., \& Dantas, A.L. (2012). Bioactive compounds and antioxidant activity in fruits of clone and ungrafted genotypes of yellow mombin tree. Food Science and Technology, 32(4), 685-691. http://dx.doi.org/10.1590/S0101-20612012005000101

Silva, F.V.G., Silva, S.M., Silva, G.C., \& Schunemann, A.P. (2013). Quality and antioxidant activity during ripening of fruits from yellow mombin (Spondias mombin L.) genotypes. Acta horticulturae, (1012), 843-848. http://dx.doi.org/10.17660/ActaHortic.2013.1012.114

Silva, L.M.R., Figueiredo, E.A.T., Ricardo, N.M.P.S., Vieira, I.G.P., Figueiredo, R.W., Brasil, I.M., \& Gomes, C.L. (2014). Quantification of bioactive compounds in pulps and by-products of tropical fruits from Brazil. Food chemistry, 143, 398-404. http://dx.doi.org/10.1016/..foodchem.2013.08.001

Sousa, S.H.B., Andrade Mattietto, R., Chisté, R. C., \& Carvalho, A.V. (2018). Phenolic compounds are highly correlated to the antioxidant capacity of genotypes of Oenocarpus distichus Mart. fruits. Food Research International, 108, 405-412. https://doi.org/10.1016/i.foodres.2018.03.056

Strohecker, R., \& Henning, H.M. (1967). Análisis de vitaminas, métodos comprobados. Madrid: Paz Montolvo.

Tiburski, J.H., Rosenthal, A., Deliza, R., Oliveira Godoy, R.L., \& Pacheco, S. (2011). Nutritional properties of yellow mombin (Spondias mombin L.) pulp. Food Research International, 44(7), 2326-2331. https://doi.org/10.1016/j.foodres.2011.03.037

Toivonen, P.M. (2016). Postharvest physiology of fruits and vegetables. In Pareek, S. Postharvest Ripening Physiology of Crops (pp. 90-121). Flórida: CRC Press. 
Vasco, C., Ruales, J., \& Kamal-Eldin, A. (2008). Total phenolic compounds and antioxidant capacities of major fruits from Ecuador. Food chemistry, 111(4), 816-823. https://doi.org/10.1016/i.foodchem.2008.04.054

Vieira, L.M., Sousa, M.S.B., Mancini-Filho, J., \& Lima, A.D. (2011). Fenólicos totais e capacidade antioxidante in vitro de polpas de frutos tropicais. Revista Brasileira de Fruticultura, 33(3), 888-897. http://dx.doi.org/10.1590/S0100-29452011005000099

Wang, S.Y., Chen, C.T., \& Wang, C.Y. (2009). The influence of light and maturity on fruit quality and flavonoid content of red raspberries. Food Chemistry, 112(3), 676-684. http://dx.doi.org/10.1016/i.foodchem.2008.06.032

Yahia, E.M., \& Ornelas-Paz, J.D.J. (2010). Chemistry, stability, and biological actions of carotenoids. In Laura, A., Alvarez-Parrilla, E., \& González-Aguilar, G. A. (Eds.). Fruit and vegetable phytochemicals: Chemistry, nutritional value and stability (pp. 177-222). Ames: Wiley-Blackwell. 